\title{
Objective hypoesthesia and pain after transabdominal preperitoneal hernioplasty: a prospective, randomized study comparing tissue adhesive versus spiral tacks
}

\author{
Lukas Brügger • Martina Bloesch • Ramin Ipaktchi • \\ Anita Kurmann • Daniel Candinas • Guido Beldi
}

Received: 18 July 2011 / Accepted: 7 October 2011/Published online: 2 November 2011

(C) Springer Science+Business Media, LLC 2011

\begin{abstract}
Background Irritation of inguinal nerves with laparoscopic hernia repair may cause chronic neuralgia and hypoesthesia. Hypoesthesia in particular is generally not assessed objectively. We objectively investigated hypoesthesia and chronic pain after transabdominal preperitoneal inguinal hernia repair (TAPP) with titanium spiral tacks (STs) compared with tissue adhesive (TA) for mesh fixation.

Methods Mesh fixation in 80 TAPP procedures was randomized to fixation with ST $(n=40)$ or TA $(n=40)$. The outcome parameters included hypoesthesia assessed with von Frey monofilaments, early postoperative and chronic pain with the visual analog scale (VAS), morbidity (surgical-site infection, hematoma/seroma, relapse of hernia, trocar hernia), and recovery time to normal activity.

Results Median (range) follow-up was 38 (13-56) months. Demographic and baseline parameters were similar in the two groups. Prevalence of hypoesthesia was significantly higher at all postoperative times in the ST group (6 weeks: 32 vs. 6\%; 6 months: 38 vs. $14 \%$; 12 months: 34 vs. $13 \%$; $13-56$ months: 32 vs. $4 \%$ ). Mean hypoesthesia scores over all time points were significantly higher in the ST group. The percentages of regions with hypoesthesia (abdominal, inguinal, or genitofemoral) following all procedures were higher in the ST group after
\end{abstract}

L. Brügger $(\bowtie) \cdot$ M. Bloesch · A. Kurmann · D. Candinas .

G. Beldi

Department of Visceral Surgery and Medicine, Bern University

Hospital, University of Bern, 3010 Bern, Switzerland

e-mail: lukas.bruegger@insel.ch

R. Ipaktchi

Department of Plastic, Reconstructive and Hand Surgery, Bern University Hospital, University of Bern, 3010 Bern, Switzerland
6 weeks (14 vs. $2 \%), 6$ months (15 vs. $5 \%)$, and $13-56$ months ( 22 vs. $4 \%$ ). The intensity of pain decreased significantly in both groups over time.

Conclusions Postoperative hypoesthesia depends on the method of mesh fixation during TAPP and is significantly reduced with TA compared with stapling.

Keywords Inguinal hernia - Laparoscopy $\cdot$ Mesh · Pain · Hypoesthesia

Compared with open tension-free mesh techniques, laparoscopic preperitoneal repair for inguinal hernia is associated with reduced incidence of surgical-site infections, earlier return to normal activity, fewer nerve injuries, and less persistent pain at comparable recurrence rates and costs [1-8]. In light of these arguments favoring the laparoscopic approach, current research is focused on technical aspects to further improve this procedure. The prevalence of long-term postoperative discomfort such as chronic inguinal or scrotal pain remains a relevant clinical problem and is still reported in up to $28.7 \%$ of patients after laparoscopic preperitoneal repair $[2,8-15]$. Mesh fixation with staples has been identified as a possible reason for nerve irritation, and osteitis pubis as the main cause of chronic pain [16-20].

Alternatively, mesh fixation with synthetic tissue adhesives (TAs) based on cyanoacrylate or fibrin glue of human origin is feasible and has been investigated in experimental and clinical studies [21-24]. Compared with mesh fixation with staples, tissue sealing is associated with reduced risk of neurovascular injury or chronic postoperative pain and earlier resumption of physical and social activities [12-15, $25,26]$.

Although nerve damage may represent the most important pathogenic factor for chronic pain, sensory 
disruptions are infrequently reported [27]. A qualitative, objective assessment of sensory dysfunction with instruments has so far been used only in an evaluation of open [28] and a comparison of open and laparoscopic hernia [29] repair, but evaluation of different fixation techniques during laparoscopic hernia repair has not been reported.

This study was designed to compare postoperative hypoesthesia when using TA or spiral tacks (STs) for intraperitoneal fixation of mesh in transabdominal preperitoneal inguinal hernia repair (TAPP). The outcome parameters of this study are an objective assessment of chronic hypoesthesia using von Frey monofilaments, postoperative pain, and surgical complications including recurrence and recovery time to normal activity.

\section{Patients and methods}

This report was prepared in accordance with the Consolidated Standards of Reporting Trials (CONSORT) statement [30]. The study was registered at clinicaltrials.gov (registration number NCT00793286).

\section{Study design and protocol}

The Ethics Committee of the University of Bern, Switzerland approved the study protocol. The design of the trial consisted of a pretreatment evaluation, randomized treatment with either tissue sealing or tack mesh fixation, in-hospital postoperative follow-up, and follow-up after discharge at 6 weeks, 6 and 12 months, and long-term follow-up.

\section{Inclusion criteria}

All patients older than 18 years presenting with inguinal hernia and fit for surgery were consecutively enrolled in the study. Operations were performed in our clinic after written consent was obtained.

\section{Exclusion criteria}

Patients with general contradictions for laparoscopy, including previous laparoscopic mesh implantation, body mass index (BMI) $>45 \mathrm{~kg} / \mathrm{m}^{2}$, pregnancy, and long-term use of immunosuppressive agents were excluded from the study.

\section{Preoperative evaluation}

All patients received a complete physical examination and standard laboratory work-up prior to surgery. Anesthesiological risk was classified according to the American Society of Anesthesiologists (ASA) classification.

\section{Randomization}

Randomization in permutated blocks of 20 was performed with sealed envelopes. The random distribution between the two groups was assessed with www.randomiza tion.com. Envelopes from patients who were excluded were discarded.

\section{Surgical technique}

Prophylactic antibiotics (amoxicillin/clavulanic acid $1.2 \mathrm{~g}$ i.v., Augmentin; GlaxoSmithKline, Münchenbuchsee, Switzerland) were given before the operation. The surgical technique for TAPP was performed as described previously [31]. Briefly, after establishing pneumoperitoneum (12 $\mathrm{mmHg}$ ), the peritoneum was incised medial to the upper iliac spine, followed by dissection until the testicular vessels, the vas deferens in male or the round ligament in female, and Cooper's ligament were identified. The hernia sac was dissected free, and a possible preperitoneal lipoma was removed. A Vypro II ${ }^{\circledR}$ (Ethicon Switzerland, Johnson \& Johnson Medical, CH-8957 Spreitenbach) prosthetic mesh $\left(10 \times 15 \mathrm{~cm}^{2}\right)$ was positioned overlapping the pubic tubercle and fixed according to randomization with either the ProTak device (5 mm titan; AutoSuture Switzerland, CH-8832 Wollerau) or Glubran ${ }^{\circledR}$ cyanoacrylate tissue sealant (G.E.M., Viareggio, Italy). Tacks were placed only on Cooper's ligament/pubic tubercle and medial of the epigastric vessels to avoid neurovascular injury. Peritoneal closure over the mesh was performed using PDS $^{\circledR} 4.0$ (Ethicon Switzerland, Johnson \& Johnson Medical, CH-8957 Spreitenbach) resorptive sutures.

Postoperative management and follow-up

The standard postoperative treatment was consistent with generally accepted principles. After discharge, no limitation to load bearing was required. Patients were seen in our outpatient clinic after 6 weeks, 6 and 12 months, and at a long-term follow-up following hernia repair. Follow-up after discharge included physical examination and an interview by an independent observer. Patients were blinded to the method of mesh fixation (single blinded).

Assessment of sensory dysfunction

For quantitative assessment of postoperative hypoesthesia, we used calibrated von Frey monofilaments (Senselab Aesthesiometer, Somedic, Stockholm, Sweden) [29]. Briefly, different pressure forces were applied with the monofilaments to the region of interest. The threshold for hypoesthesia was defined as the least pressure that elicited a sensation. The score for hypoesthesia was calculated by 
adding intensity values of hypoesthesia as measured by the von Frey monofilaments. The mechanical pain threshold for allodynia was defined as the lowest force that elicited a sensation of pain or discomfort.

Pain management and assessment

All patients received standardized perioperative pain management according to established postoperative management protocols in our hospital. Patients received paracetamol and opioids in a weight-dependent dosage. After discharge, pain intensity was assessed at the defined time points with a visual analog scale (VAS) of $10 \mathrm{~cm}$. Chronic postoperative pain was defined as VAS $\geq 3$.

Endpoints of the study

The primary outcome parameter was hypoesthesia (von Frey monofilaments). Secondary outcome parameters included postoperative pain (VAS), morbidity (surgicalsite infection, hematoma/seroma, relapse of hernia, trocar hernia), and recovery time to normal activity. All points of interest and all complications or adverse reactions were documented in the patient record form.

Statistical analysis

Statistical analysis was performed per protocol. Based on the premise of improving the prevalence of postoperative hypoesthesia from $45 \%$ with ST to $15 \%$ with TA for mesh fixation ( $\alpha$ set at 0.05 , power of $80 \%$ ) we calculated that 40 patients were required for each arm of the study. Chisquare or Fisher's exact analyses as appropriate were used to compare proportions and the relationships among categorical data. The Wilcoxon rank-sum test for differences in medians was used for quantitative variables. Repeatedmeasures analysis of variance (ANOVA) of means was performed to assess the time effect and group effect for quantitative variables. All tests were performed bilaterally with a 5\% significance threshold. The NCSS/PASS software package (NCSS, Kaysville, UT, USA) was used for all calculations.

\section{Results}

A flowchart of participants through each stage of the trial is depicted in Fig. 1. Eligible patients were recruited between August 2004 and November 2007. After discharge, all participants were followed up in our outpatient clinic at 6 weeks, 6 and 12 months, and a median of 38 (range 13-56) months after surgery.
Patient characteristics and surgical details

Baseline characteristics of the patients are summarized in Table 1. No intraoperative complications occurred in either group. Operative details, surgical perioperative morbidity, and follow-up details are presented in Table 2.

Postoperative hypoesthesia and allodynia

After mesh fixation with STs, hypoesthesia was noted significantly more often at all postoperative time points compared with the TA group (Table 3). After 12 months, the median hypoesthesia score was 42 (range 16-135) in the tack fixation group compared with 16 (range 11-52) in the TA group $(P$-value $=0.045)$. As shown in Fig. 2, analysis of repeated measurements of all procedures also revealed a significantly higher score for hypoesthesia in the ST group $\left(P\right.$-value $\left._{\text {groups }}=0.020\right)$, and the hypoesthesia score did not change significantly over time $\left(P\right.$-value $\left._{\text {time }}=0.740\right)$. The percentages of regions with hypoesthesia (abdominal, inguinal, or genitofemoral) after all procedures in the two treatment groups are shown in Fig. 3. Percentages were higher in the ST group at 6 weeks (14 vs. 2\%), 6 months (15 vs. 5\%), and 13-56 months ( 22 vs. $4 \%$; all $P$-values $<0.05$ ) compared with the TA group.

The median hypoesthesia score in the ST group was significantly higher in patients with pain compared with patients without pain after 6 weeks (20, range 0-390 versus 0 , range $0-149, P$-value $=0.046)$. No differences were found at the other time points and in the TA group. Incidences of allodynia did not differ between the ST group and the TA group at the different postoperative time points (6 weeks: 5.9 vs. $8.3 \%$; 6 months: 6.1 vs. $11.4 \%$; 12 months: 6.3 vs. $6.5 \%$; $13-56$ months: 3.5 vs. $0 \%$ ). No differences in intensity scores of allodynia between the treatment groups were found (data not shown).

Postoperative pain assessment

Analysis of repeated measurements demonstrated a significant decrease in pain over time $\left(P\right.$-value $\left._{\text {time }}<0.001\right)$. However, no difference in intensity (VAS) between the treatment groups was found (Fig. 4). The percentages of painful areas (abdominal, inguinal, genitofemoral) were significantly higher in the tack fixation group at the early time points ( 6 weeks, 26 vs. $11 \%$; 6 months, 23 vs. $11 \%$; all $P$-values $<0.05)$, whereas no differences were found at later time points (Fig. 5). No statistically significant differences in prevalence of postoperative pain or VAS scores were found between the two fixation methods (Table 4). 
Fig. 1 Experimental flowchart

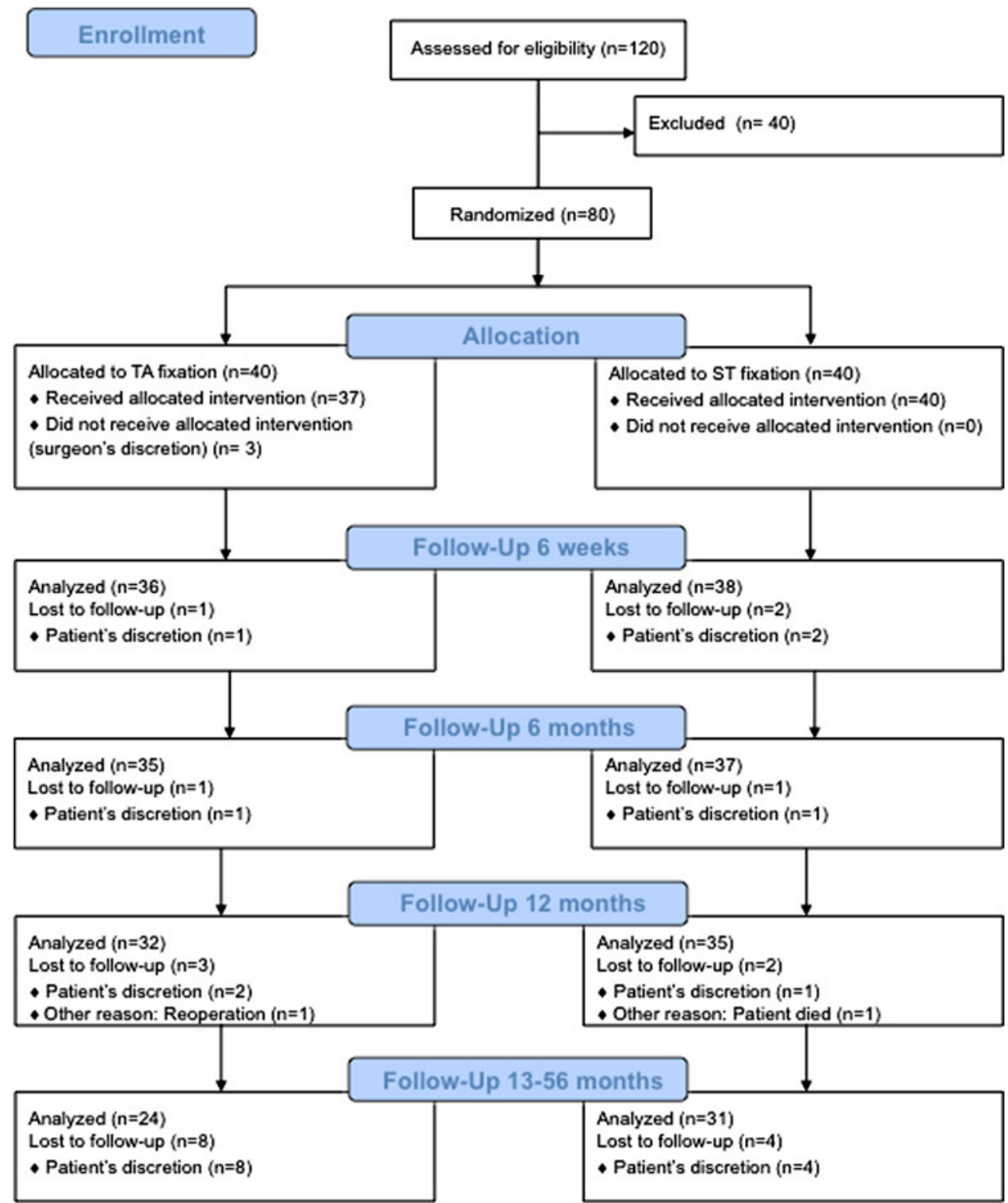

tissue adhesive (TA); spiral tacks (ST)

\section{Discussion}

In this study, we demonstrated higher incidence and wider distribution over different regions of hypoesthesia after use of STs compared with TA mesh fixation for TAPP. This effect was observed at early and late time points.

The results from this study objectively reveal that numbness is a common late consequence of laparoscopic groin hernia repair, occurring as shown before in up to $67 \%$ of patients [29, 32-34]. In our current study, we also demonstrated that hypoesthesia is not reversible during long-term follow-up. Conversely, postoperative pain decreased over time. Therefore, prevention of hypoesthesia with an adequate, suitable operation technique is of clinical importance. Hyposensibility in patients who underwent laparoscopic hernia repair with tacks for mesh fixation was significantly associated with postoperative pain after 6 weeks. A relationship between pain and sensory impairment has been postulated [29, 32]. However, at later time points and in the TA group, this correlation did not reach statistical significance in our series. Therefore, factors other than nerve damage may be involved in the development of chronic post-herniotomy pain [28]. In our study, incidences of chronic postoperative pain were noted in less than $10 \%$ of patients during long-term follow-up. Previous studies have reported chronic pain in up to $28.7 \%$ of patients after laparoscopic hernia repair [2, 8-15]. 
Table 1 Baseline demographic and clinical characteristics $(n=80)$

\begin{tabular}{llll}
\hline Characteristic & TA & STs & $P$ value \\
\hline Sex ratio (M:F) & $37: 0$ & $39: 1$ & 0.333 \\
Age (years) & $57.3(20.9-82.5)$ & $59.9(19.9-82.2)$ & 0.617 \\
ASA score $>2$ & $1 / 37(3 \%)$ & $24.7(18.4-32.6)$ & 0.057 \\
BMI $\left(\mathrm{kg} / \mathrm{m}^{2}\right)$ & $24.8(20.9-33.7)$ & & 0.275 \\
Type of hernia & & $20(50 \%)$ & $17(43 \%)$ \\
Lateral & $22(60 \%)$ & $1(3 \%)$ & $1(3 \%)$ \\
Medial & $14(38 \%)$ & $1(3 \%)$ \\
Femoral & - & $18 / 40(45 \%)$ \\
Mixed & - & & 0.686 \\
Hernia incipiens & $1(3 \%)$ & $13(33 \%)$ \\
Bilateral hernia & $14 / 37(38 \%)$ & $15(38 \%)$ \\
Diameter of hernia & $12(32 \%)$ & $12(30 \%)$ \\
$<1.5 \mathrm{~cm}$ & $19(51 \%)$ & $3(0-60)$ \\
$1.5-3 \mathrm{~cm}$ & $6(16 \%)$ & $2(0-48)$ & 0.644 \\
$>3 \mathrm{~cm}$ & & 0.373 \\
Symptoms history (months) &
\end{tabular}

Quantitative data are given as median and range

ASA American Society of Anesthesiologists, BMI body mass index

The recurrence rate, time to normal physical activity, and perioperative and late morbidity did not differ between the treatment groups. These findings are consistent with results from previous clinical trials examining mesh fixation with tacks versus TA [12-15, 25, 26]. In these studies, both fixation techniques have been shown to be adequate for preventing early postoperative mesh dislocation without increasing the recurrence rate.

Our study has several important strengths. First, the design was a blinded, randomized controlled trial, providing a high level of evidence to the conclusions. Second, the median follow-up time of more than 3 years is longer than in other studies comparing different fixation techniques $[12-15,25,26]$. Third, in our investigation, instruments were used for an objective assessment of sensory function. Just a few previous studies contain data reporting examination of sensory function [27]. Only a precise, objective assessment of hypoesthesia allows comparison between different studies and will therefore result in a reduction in long-term discomfort.

A limitation of this study is the restricted sample size that did not allow detection of differences in recurrence rates. However, the sample size met the requirements to demonstrate differences in sensory disruptions, which was our primary endpoint.

In conclusion, this study represents the first examination with an objective neurophysiological sensory function test after different types of mesh fixation in laparoscopic inguinal hernia repair. No differences were found in the clinical incidence or intensity of postoperative pain.
Table 2 Operative details, morbidity, and follow-up

\begin{tabular}{llll}
\hline Characteristic & TA & STs & $P$ value \\
\hline Operation time (min) & $90(45-180)$ & $85(55-180)$ & 0.872 \\
In-hospital stay (days) & $5(2-9)$ & $5(2-9)$ & 0.554 \\
Time to activity/work (days) & $19(5-35)$ & $21(7-56)$ & 0.908 \\
Perioperative morbidity & $3 / 37(8 \%)$ & $1 / 40(3 \%)$ & 0.340 \\
Surgical-site infection & $0 / 37(0 \%)$ & $0 / 40(0 \%)$ & - \\
Hematoma/seroma & $3 / 37(8 \%)$ & $1 / 40(3 \%)$ & 0.340 \\
Late morbidity & $2 / 32(6 \%)$ & $1 / 35(3 \%)$ & 0.603 \\
Trocar hernia & $1 / 32(3 \%)$ & $0 / 35(0 \%)$ & 0.478 \\
Recurrence & $2 / 32(6 \%)$ & $1 / 35(3 \%)$ & 0.603 \\
Follow-up (months) & $45(14-56)$ & $37(13-51)$ & 0.070 \\
\hline
\end{tabular}

Quantitative data are given as median and range

Table 3 Prevalence of postoperative hypoesthesia score $>0$

\begin{tabular}{llll}
\hline Time point & TA & STs & $P$ value \\
\hline Preoperative & $11 / 37(30 \%)$ & $10 / 40(25 \%)$ & 0.799 \\
6 weeks & $2 / 36(6 \%)$ & $12 / 38(32 \%)$ & 0.006 \\
6 months & $5 / 35(14 \%)$ & $14 / 37(38 \%)$ & 0.032 \\
12 months & $4 / 32(13 \%)$ & $12 / 35(34 \%)$ & 0.047 \\
$13-56$ months & $1 / 24(4 \%)$ & $10 / 31(32 \%)$ & 0.010 \\
\hline
\end{tabular}

However, hypoesthesia was more common and more severe after use of tacks for mesh fixation. In contrast to postoperative chronic pain, the intensity of hypoesthesia did not significantly decrease over time after tack fixation. Therefore, use of TA, which is associated with fewer 


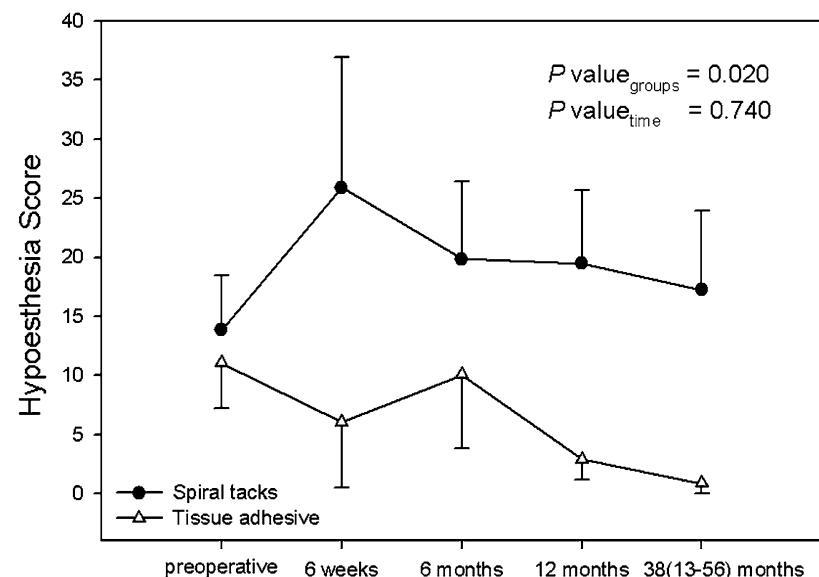

Fig. 2 Group and time effect of the hyposensibility score comparing the TA and the ST groups (repeated-measures ANOVA). Values are given as means, and bars indicate standard error of the mean

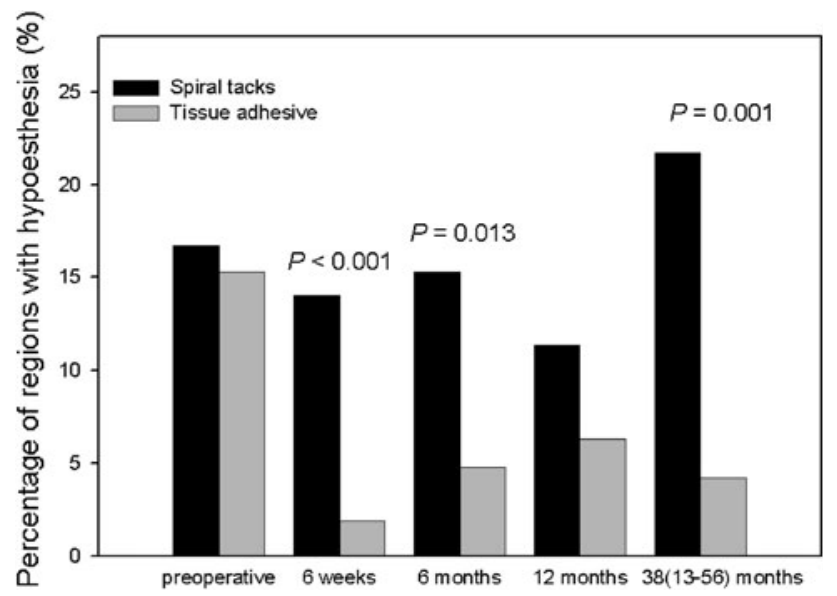

Fig. 3 Percentage of regions with hypoesthesia (abdominal, inguinal, or genitofemoral) of all procedures in the groups with tissue sealing and STs

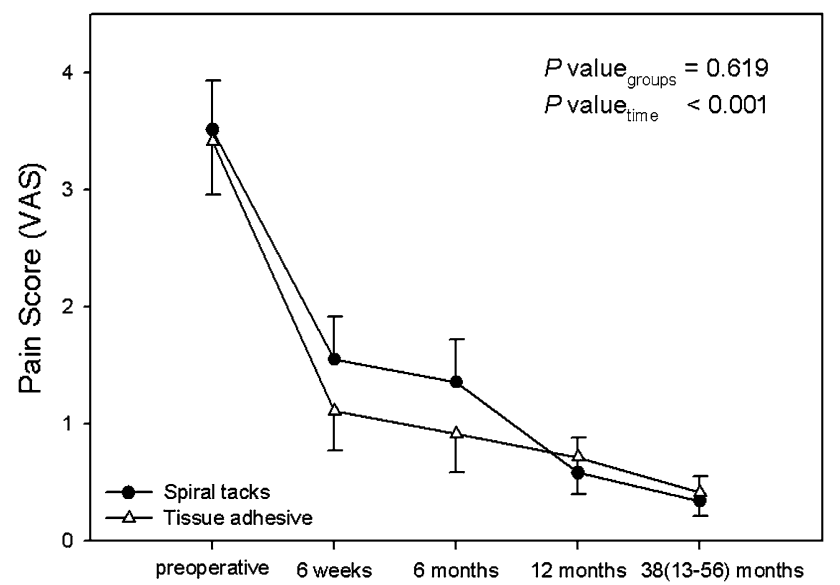

Fig. 4 Group and time effect of pain score (VAS) comparing the TA and the ST groups. Values are given as means, and bars indicate standard error of the mean

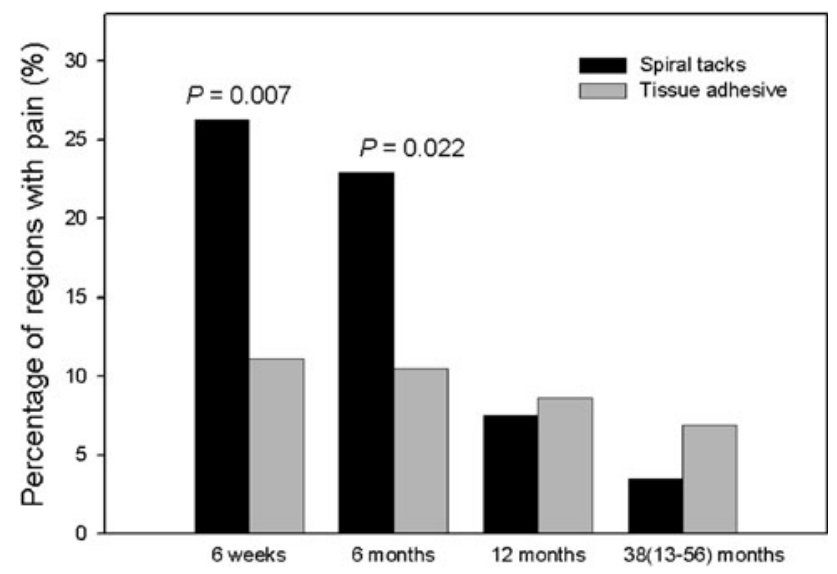

Fig. 5 Percentage of regions with pain (abdominal, inguinal, or genitofemoral) in the groups with tissue sealing and STs

Table 4 Prevalence of chronic postoperative pain and postoperative pain scores (VAS) of patients with chronic pain

\begin{tabular}{llll}
\hline Time point & TA & STs & $P$ value \\
\hline Chronic pain $(n)$ & & & \\
Preoperative & $19 / 34(56 \%)$ & $26 / 39(67 \%)$ & 0.345 \\
6 weeks & $8 / 36(22 \%)$ & $10 / 38(26 \%)$ & 0.682 \\
6 months & $6 / 35(17 \%)$ & $9 / 37(24 \%)$ & 0.566 \\
12 months & $5 / 32(16 \%)$ & $3 / 35(9 \%)$ & 0.465 \\
13-56 months & $2 / 24(8 \%)$ & $2 / 31(7 \%)$ & 1.000 \\
Total VAS score $($ median, range) & & \\
Preoperative & $5(3-9)$ & $5(3-8)$ & 0.782 \\
6 weeks & $4(3-7)$ & $5(3-8)$ & 0.820 \\
6 months & $6(3-7)$ & $5(3-8)$ & 0.903 \\
12 months & $4(3-7)$ & $3(3-10)$ & 0.645 \\
13-56 months & $3(3-3)$ & $4(3-5)$ & 0.317 \\
\hline
\end{tabular}

sensory disruptions, for mesh fixation is important for laparoscopic treatment of inguinal hernias.

Acknowledgements We thank Brigitte Wanner for meticulous data collection and Dr. Stephan Vorburger for advice with statistical analysis. This study was supported by a grant from Anklin AG, 4102 Binningen, Switzerland.

Disclosures Authors Lukas Brügger, Martina Boesch, Ramin Ipaktchi, Anita Kurmann, Daniel Candinas, and Guido Beldi have no conflicts of interest or financial ties to disclose.

\section{References}

1. EU Hernia Trialists Collaboration (2002) Repair of groin hernia with synthetic mesh: meta-analysis of randomized controlled trials. Ann Surg 235:322-332

2. McCormack K, Scott NW, Go PM, Ross S, Grant AM (2003) Laparoscopic techniques versus open techniques for inguinal hernia repair. Cochrane Database Syst Rev CD001785 
3. Pokorny H, Klingler A, Schmid T, Fortelny R, Hollinsky C, Kawji R, Steiner E, Pernthaler H, Fugger R, Scheyer M (2008) Recurrence and complications after laparoscopic versus open inguinal hernia repair: results of a prospective randomized multicenter trial. Hernia 12:385-389

4. Schmedt CG, Sauerland S, Bittner R (2005) Comparison of endoscopic procedures vs. Lichtenstein and other open mesh techniques for inguinal hernia repair: a meta-analysis of randomized controlled trials. Surg Endosc 19:188-199

5. Memon MA, Cooper NJ, Memon B, Memon MI, Abrams KR (2003) Meta-analysis of randomized clinical trials comparing open and laparoscopic inguinal hernia repair. $\mathrm{Br} \mathrm{J}$ Surg 90:1479-1492

6. Butters M, Redecke J, Koninger J (2007) Long-term results of a randomized clinical trial of shouldice, lichtenstein and transabdominal preperitoneal hernia repairs. Br J Surg 94:562-565

7. Eklund A, Carlsson P, Rosenblad A, Montgomery A, Bergkvist L, Rudberg C (2010) Long-term cost-minimization analysis comparing laparoscopic with open (lichtenstein) inguinal hernia repair. Br J Surg 97:765-771

8. Eklund A, Montgomery A, Bergkvist L, Rudberg C (2010) Chronic pain 5 years after randomized comparison of laparoscopic and lichtenstein inguinal hernia repair. Br J Surg 97: 600-608

9. The MRC Laparoscopic Groin Hernia Trial Group (1999) Laparoscopic versus open repair of groin hernia: a randomised comparison. Lancet 354:185-190

10. Hindmarsh AC, Cheong E, Lewis MP, Rhodes M (2003) Attendance at a pain clinic with severe chronic pain after open and laparoscopic inguinal hernia repairs. Br J Surg 90:1152-1154

11. Kumar S, Wilson RG, Nixon SJ, Macintyre IM (2002) Chronic pain after laparoscopic and open mesh repair of groin hernia. $\mathrm{Br}$ J Surg 89:1476-1479

12. Lau H (2005) Fibrin sealant versus mechanical stapling for mesh fixation during endoscopic extraperitoneal inguinal hernioplasty: a randomized prospective trial. Ann Surg 242:670-675

13. Lovisetto F, Zonta S, Rota E, Mazzilli M, Bardone M, Bottero L, Faillace G, Longoni M (2007) Use of human fibrin glue (Tissucol) versus staples for mesh fixation in laparoscopic transabdominal preperitoneal hernioplasty: a prospective, randomized study. Ann Surg 245:222-231

14. Schwab R, Willms A, Kroger A, Becker HP (2006) Less chronic pain following mesh fixation using a fibrin sealant in TEP inguinal hernia repair. Hernia 10:272-277

15. Topart $P$, Vandenbroucke F, Lozac'h $P$ (2005) Tisseel versus tack staples as mesh fixation in totally extraperitoneal laparoscopic repair of groin hernias: a retrospective analysis. Surg Endosc 19:724-727

16. Andrew DR, Gregory RP, Richardson DR (1994) Meralgia paraesthetica following laparoscopic inguinal herniorrhaphy. Br J Surg 81:715

17. Broin EO, Horner C, Mealy K, Kerin MJ, Gillen P, O’Brien M, Tanner WA (1995) Meralgia paraesthetica following laparoscopic inguinal hernia repair. An anatomical analysis. Surg Endosc 9:76-78

18. Stark E, Oestreich K, Wendl K, Rumstadt B, Hagmuller E (1999) Nerve irritation after laparoscopic hernia repair. Surg Endosc $13: 878-881$
19. Beattie GC, Kumar S, Nixon SJ (2000) Laparoscopic total extraperitoneal hernia repair: mesh fixation is unnecessary. J Laparoendosc Adv Surg Tech A 10:71-73

20. Poobalan AS, Bruce J, Smith WC, King PM, Krukowski ZH, Chambers WA (2003) A review of chronic pain after inguinal herniorrhaphy. Clin J Pain 19:48-54

21. Hollinsky C, Gobl S (1999) Bursting strength evaluation after different types of mesh fixation in laparoscopic herniorrhaphy. Surg Endosc 13:958-961

22. Katkhouda N, Mavor E, Friedlander MH, Mason RJ, Kiyabu M, Grant SW, Achanta K, Kirkman EL, Narayanan K, Essani R (2001) Use of fibrin sealant for prosthetic mesh fixation in laparoscopic extraperitoneal inguinal hernia repair. Ann Surg 233:18-25

23. Fortelny RH, Petter-Puchner AH, Walder N, Mittermayr R, Ohlinger W, Heinze A, Redl H (2007) Cyanoacrylate tissue sealant impairs tissue integration of macroporous mesh in experimental hernia repair. Surg Endosc 21:1781-1785

24. Jourdan IC, Bailey ME (1998) Initial experience with the use of $\mathrm{N}$-butyl 2-cyanoacrylate glue for the fixation of polypropylene mesh in laparoscopic hernia repair. Surg Laparosc Endosc 8:291-293

25. Ceccarelli G, Casciola L, Pisanelli MC, Bartoli A, Di Zitti L, Spaziani A, Biancafarina A, Stefanoni M, Patriti A (2008) Comparing fibrin sealant with staples for mesh fixation in laparoscopic transabdominal hernia repair: a case control-study. Surg Endosc 22:668-673

26. Olmi S, Scaini A, Erba L, Guaglio M, Croce E (2007) Quantification of pain in laparoscopic transabdominal preperitoneal (TAPP) inguinal hernioplasty identifies marked differences between prosthesis fixation systems. Surgery 142:40-46

27. Aasvang E, Kehlet H (2005) Chronic postoperative pain: the case of inguinal herniorrhaphy. Br J Anaesth 95:69-76

28. Mikkelsen T, Werner MU, Lassen B, Kehlet H (2004) Pain and sensory dysfunction 6 to 12 months after inguinal herniotomy. Anesth Analg 99:146-151

29. Beldi G, Haupt N, Ipaktchi R, Wagner M, Candinas D (2008) Postoperative hypoesthesia and pain: qualitative assessment after open and laparoscopic inguinal hernia repair. Surg Endosc 22:129-133

30. Begg C, Cho M, Eastwood S, Horton R, Moher D, Olkin I, Pitkin R, Rennie D, Schulz KF, Simel D, Stroup DF (1996) Improving the quality of reporting of randomized controlled trials. The CONSORT statement. JAMA 276:637-639

31. Gerber S, Hammerli PA, Glattli A (2000) Laparoscopic transabdominal preperitoneal hernioplasty. Evaluation of complications due to transabdominal approach. Chirurg 71:824-828

32. Cunningham J, Temple WJ, Mitchell P, Nixon JA, Preshaw RM, Hagen NA (1996) Cooperative hernia study. Pain in the postrepair patient. Ann Surg 224:598-602

33. Gillion JF, Fagniez PL (1999) Chronic pain and cutaneous sensory changes after inguinal hernia repair: comparison between open and laparoscopic techniques. Hernia 3:5

34. Heikkinen T, Bringman S, Ohtonen P, Kunelius P, Haukipuro K, Hulkko A (2004) Five-year outcome of laparoscopic and Lichtenstein hernioplasties. Surg Endosc 18:518-522 